\title{
A Novel Homozygous Missense Mutation of WEE2 Causes Female Infertility Characterized by Fertilization Failure
}

\section{Wenwen Liu ( $D$ 779760636@qq.com )}

Nanjing Drum Tower Hospital: Nanjing University Medical School Affiliated Nanjing Drum Tower Hospital https://orcid.org/0000-0001-8877-6601

\section{Guijun Yan}

Nanjing Drum Tower Hospital: Nanjing University Medical School Affiliated Nanjing Drum Tower Hospital

\section{Ningyuan Zhang}

Nanjing Drum Tower Hospital: Nanjing University Medical School Affiliated Nanjing Drum Tower Hospital

Na Kong

Nanjing Drum Tower Hospital: Nanjing University Medical School Affiliated Nanjing Drum Tower Hospital

\section{Min Wu}

Nanjing Drum Tower Hospital: Nanjing University Medical School Affiliated Nanjing Drum Tower Hospital

\section{Junshun Fang}

Nanjing Drum Tower Hospital: Nanjing University Medical School Affiliated Nanjing Drum Tower Hospital

\section{Haixiang Sun}

Nanjing Drum Tower Hospital: Nanjing University Medical School Affiliated Nanjing Drum Tower Hospital

\section{Research}

Keywords: Fertilization failure, WES , WEE2, missense mutation, infertility

Posted Date: July 12th, 2021

DOI: https://doi.org/10.21203/rs.3.rs-650191/v1

License: (c) (1) This work is licensed under a Creative Commons Attribution 4.0 International License. Read Full License 

A novel homozygous missense mutation of WEE2 causes female infertility characterized by fertilization failure

Wenwen Liu, Guijun Yan, Ningyuan Zhang, Na Kong, Min Wu, Junshun Fang, Haixiang Sun*

Authors' information

Wenwen Liu, 779760636@qq.com, Reproductive Medicine Center, The Affiliated Drum Tower Hospital of Nanjing University Medical School, Nanjing 210008, People's Republic of China.

Guijun Yan, yanguijun55@163.com, Reproductive Medicine Center, The Affiliated Drum Tower Hospital of Nanjing University Medical School, Nanjing 210008, People's Republic of China.

Ningyuan Zhang, katty_ny@163.com, Reproductive Medicine Center, The Affiliated Drum Tower Hospital of Nanjing University Medical School, Nanjing 210008, People's Republic of China.

Na Kong, xtalkn@163.com, Reproductive Medicine Center, The Affiliated Drum Tower Hospital of Nanjing University Medical School, Nanjing 210008, People's Republic of China.

Min Wu, 973312733@qq.com, Reproductive Medicine Center, The Affiliated Drum Tower Hospital of Nanjing University Medical School, Nanjing 210008, People's Republic of China.

Junshun Fang, fangjunshun@,163.com, Reproductive Medicine Center, The Affiliated Drum Tower Hospital of Nanjing University Medical School, Nanjing 210008, People's Republic of China.

Haixiang Sun, stevensunz@163.com, Reproductive Medicine Center, The Affiliated Drum Tower Hospital of Nanjing University Medical School, Nanjing 210008, People's Republic of China. *Corresponding author 
A novel homozygous missense mutation of WEE2 causes female infertility characterized by fertilization failure

Wenwen Liu ${ }^{1}$, Guijun Yan ${ }^{1}$, Ningyuan Zhang ${ }^{1}$, Na Kong ${ }^{1}$, Min Wu ${ }^{1}$, Junshun Fang ${ }^{1}$, Haixiang $\operatorname{Sun}^{1 *}$

${ }^{1}$ Reproductive Medicine Center, The Affiliated Drum Tower Hospital of Nanjing University Medical School, Nanjing 210008, People's Republic of China.

*Corresponding author

Abstract

Purpose: To investigate the genetic cause of infertility in a female patient due to repeated fertilization failure.

Methods: Whole exome sequencing was performed to obtain the candidate mutation. Sanger sequencing was used to identify the mutation of the proband and other family members. SIFT, Polyphen-2, and Mutation Taster were used to predict the pathogenicity of mutations. The online software Arpeggio and the mCSM online service were used to analyze the effect of the mutation on protein structure and stability.

Results: We identified a novel homozygous missense mutation c.T1199A:p.L400Q (Leu400Gln) in WEE2 gene in a female proband with infertility caused by fertilization failure.

Conclusions: We discovered a novel homozygous missense mutation c.T1199A:p.L400Q (Leu400Gln) of the WEE2 gene in an infertile female whose oocytes had undergone complete fertilization failure, either after ICSI or RICSI. Our findings extend the mutant spectrum of WEE2, a genetic cause for fertilization failure, and provide a theoretical basis for clinical diagnosis of the pathogenic causes of infertility.

Keywords: Fertilization failure, WES, WEE2, missense mutation, infertility

Introduction

Successful fertilization of sperm and oocytes is the basis of sexual reproduction, which is a highly regulated process[1]. Abnormalities in this process can lead to failure of fertilization in humans[2]. Clinically, sperm and oocyte morphology are normal, but fertilization always fails, even when oocytes are remedied by in vitro fertilization (IVF) or intracytoplasmic sperm injection (ICSI)[3]. Several homozygous and biallelic mutations in WEE2(WEE1 homolog 2, also known as WEE1B) have 
been reported to be associated with fertilization failure[4-11]. WEE2 (MIM: 614084) gene encoding Wee2 protein belongs to the Wee kinase protein family[12], which is specifically expressed in oocytes and highly conserved in various species. Wee2 is a key regulator of oocyte meiosis and plays an important role in different stages of meiosis. In the germinal vesicle (GV) stage of mouse oocytes, Wee2 inhibits maturation promoter factor (MPF) by inhibiting phosphorylation of Cell Division Cycle $2(\mathrm{Cdc} 2$, also known as Cyclin Dependent Kinase 1 ,CDK1) to maintain meiotic arrest[13]. In the metaphase II (MII) stage, Wee2 is required for MII exit and pronuclei formation, which is related to the inhibition of $\mathrm{Cdc} 2$ phosphorylation by Wee2, leading to MPF inactivation[14]. In the present study, we identified a novel homozygous missense mutation (c.T1199A:p.L400Q) of the WEE2 gene in a patient who had repeated fertilization failures after both rescue intracytoplasmic sperm injection(RICSI) and ICSI using whole exome sequencing (WES) analysis and Sanger sequcecing. And this new finding extends WEE2 mutations that are genetically responsible for repeated fertilization failure due to oocyte dysfunction.

Materials and methods

Clinical Samples

In this study, the couple infertile due to failed fertilization came from a nonconsanguineseous family and was diagnosed as primary infertility in the Nanjing Drum Tower Staff. The proband was a 37-year-old woman with a 6-year history of primary infertility. She had received intrauterine insemination (IUI) twice in our hospital in 2015 and ovulated without becoming pregnant. Her husband's total sperm motility was $65.8 \%$, with a normal rate of $8.2 \%$. The couple had normal karyotypes. The proband's two sisters and one brother were fertile, having two, three and two children, respectively. Blood samples were obtained from this affected patient, and saliva from other consanguinity was collected using a salivary collector (Norgen's Saliva DNA Collection, RU49000). This study has been approved by the Ethics Committee of Nanjing Drum Tower Staff, and we have obtained the informed consent of all 
participants in this study.

Genomic DNA Extraction and Whole-Exome Sequencing

Genomic DNA (gDNA) is extracted from a blood sample (Magnetic Blood Genomic DNA Kit, TIANGEN, DP329) and a saliva sample (Norgen's Saliva DNA Isolation Kit, RU45400) according to standard procedures, respectively. The gDNA which from the patient was used for whole-exome sequencing, and that from consanguinity was used for Sanger sequencing. Whole exome was captured with Twist Bioscience NGS Targeted Enrichment Kit and sequenced using the Illumina NovaSeq 6000 platform by the Genergy Bio-technology at Shanghai. The sequencing depth of WES was 50X.

\section{Data Analysis}

Sequencing data was stored in Fastaq (FQ) file format. To identify candidate causative mutations, we removed mutations that were not on exons and splices, and then filtered out the polymorphisms with allele frequencies greater than $1 \%$ in the Exome Aggregation Consortium (ExAC) and the 1000 Genomes Project Database (1000g2015aug_eas). The identified mutations were analyzed by SIFT, Polyphen-2 and Mutation Taster for their effects on the proteins. The conservation statuses of the mutation sites were estimated by UGENE. We used online software Arpeggio[15] and mCSM online service[16] to analyze the effect of the mutation on the structure and stability of Wee2 protein.

\section{Sanger Sequencing}

The identified variation revealed by WES sequencing was validated by Sanger sequencing in proband and her parents and siblings. Sanger sequencing was performed by polymerase chain reaction (PCR) according to the kit instructions (Phanta ${ }^{\circledR}$ Max Super-Fidelity DNA Polymerase, Vazyme, P505) and then sequencing of the PCR product on 3730XL Genetic Analyzer (Applied Biosystems) by BGI (The Beijing Genomics Institute). Sequences of primers for PCR were designed online using NCBI's "Primer-Blast" online design tool and synthesized by GenScript. The forward primer 
sequence was 5'-GCTGTTCAGAAGCGATGTGA-3' and the reverse primer sequence was 5'-TTCTGAATGGGAAGAGCGGT-3' for identifying c.T1199A site mutation of WEE2. The software SnapGene was used to compare and analyze the sequence sequenced by Sanger with the full-length sequence of human WEE2 gene, so as to determine the mutation site and whether there were mutations in her parents and siblings.

Results

Clinical characteristics of the female proband

In 2015, the proband underwent IUI twice in our hospital, and ovulated but did not become pregnant. Then in 2019, after the combination of the ultra-long scheme with RICSI attempt and the combination of the progestin-primed ovarian stimulation (PPOS) scheme with ICSI attempt were carried out in our hospital twice respectively, but the cycles were cancelled both times due to no transplantable embryos were obtained (Table 1). In the first IVF cycle, a total of 18 oocytes were obtained, including 14 oocytes at the MII stage and 4 oocytes with two polar bodies. However, none of the oocytes were successfully fertilized after the implementation of RICSI though 5 out of 14 oocytes formed 2PN (pronucleus), and 1 out of 14 oocytes formed 3PN. In the second IVF cycle, a total of 7 oocytes were obtained, all of which were in the MII stage. However, after ICSI, none of the oocytes were successfully fertilized (Figure 1).

Identification of the novel homozygous mutation in WEE2

In our current case, we identified a novel homozygous mutation c.T1199A:p.L400Q (Leu400Gln) in WEE2 by WES, which had not previously been reported. The allele frequency of this mutation was not found in the ExAC database or the1000g2015aug_eas datebase and the effect of this mutation on the protein sequence was predicted by SIFT, PolyPhen-2, and Mutation Taster software to be pathogenic (Table 2). Sanger sequencing showed that the proband had one allele of this mutation from her mother, while the other allele was a spontaneous mutation because her father 
had no mutation of either allele, and her two sisters were carriers of the mutation and were unaffected like her mother (Figure 2). The identified missense mutation was confirmed to be conserved among different species by UGENE analysis and was located in the PKinase domain of Wee2 (Figure 3). We used the online software Arpeggio to analyze the interatomic interactions in mutation of leucine at position 400 to glutamine of protein, the results showed that compared with the wild-type mutant, the six hydrophobic bonds that maintained the stability of the protein disappeared (Figure 4). Instead, a hydrogen bond was formed, indicating that there may be unstable factors in the structure of the mutant (See red dashed box). Furthermore, we used the mCSM online service to predict the effect of mutations in protein, and the results showed that mutations are not conducive to protein stability, which is consistent with the previous analysis of factors that cause instability due to changes in protein interatomic interactions before and after mutations.

\section{Discussion}

A zygote is produced by fertilization of an oocyte with a sperm and is the basis of sexual reproduction. Fertilization plays a central role in the continuation of the gamete to the embryo. The fate of embryo development depends on how fertilization develops, so the assessment of the fertilization status of oocytes is an important part of IVF treatment[17]. Successful fertilization involves several successive dynamic events, including oocytes maturation, sperm-oocytes binding, and pronucleus formation. Abnormality in any of these processes, such as sperm-oocyte binding or oocyte exit from meiotic arrest, will result in fertilization failure[18]. The combination of ICSI during in vitro fertilization usually remedies the majority of fertilization failures, but complete fertilization fails still occurs in $1-3 \%$ of oocytes, of which $60-70 \%$ are due to defective oocyte activation caused by the abnormal exit of meiotic arrest[19]. Maturation of oocytes is regulated by MPF, a heterodimer composed of Cdc2 and cyclin B. In the process of follicle maturation, cAMP in somatic cells of antral follicles around oocytes is constantly produced and transferred to oocytes, leads to WEE1B 
phosphorylation and activation. This activation will inhibit the phosphorylation of Cdc2 through the inactivation of $\mathrm{Cdc} 25$, thus leading to the continuous inactivation of MPF. This inactivation allows oocytes to remain in meiotic arrest[20]. Studies in mice showed that when Wee 2 expression decreased, the activity of MPF in oocytes increased, leading to failure of both MII exit and pronucleus formation, resulting in infertility in mice[14]. Recently, several WEE2 mutations have been identified as associated with fertilization failure in human oocytes[4-11].

In this study, we identified a new homozygous missense mutation c.T1199A:p.L400Q in the WEE2 gene in a proband with infertility. The proband underwent two IVF combined ICSI or RICSI procedures in our hospital, but she failed to obtain successfully fertilized zygotes in the end although $2 \mathrm{PN}$ formed during the first cycle. This mutation is highly conserved in multiple species and located in the Pkinase domain of Wee2 protein. In a study reported by Sang[4], cytological verification of the functional influence of c.700G $>$ C:p.D234H (p.Asp234His) showed that the mutation caused a significant reduction in phosphorylation of Wee2. The mutation identified in our study is also in the domain of PKinase. In addition, using the software Arpeggio, we predicted that the hydrophobic bond between atoms that previously kept the protein stable at the mutation site disappeared and instead an additional hydrogen bond formed. As predicted by $\mathrm{mCSM}$, this mutation was detrimental to the stability of the protein. So, it is inferred that the mutation may lead to reduced Wee 2 phosphorylation, reduced inhibition of MPF, and affected meiosis exit of oocytes, leading to fertilization failure. In our present study, $2 \mathrm{~PB}$ formation was still observed in some oocytes of the proband, although fertilization failed. Similar phenomenon was also observed in the previous study $[4,5]$. Considering that Wee2 is not the only component of MPF, when the function of Wee2 is abnormal, there may be other ways to regulate the extrusion of the second polar body, which needs to be further studied.

In conclusion, we captured a novel homozygous missense mutation of WEE2 (c.T1199A:p.L400Q) by WES in an infertile patient who failed to fertilization after two IVF treatments in our hospital in combination with RICSI and ICSI, respectively. The 
mutation was verified by Sanger sequencing on other members of the family. The mother of the proband was the heterozygous carrier of the mutation, while the father did not carry the mutation. The fertility of the two heterozygous sisters carrying the mutation was normal. According to software prediction analysis, this missense mutation is pathogenic, and due to the mutation of leucine to glycine, the hydrophobic bond between atoms is lost and an additional hydrogen bond is added, and the stability of the protein is decreased. The above evidence indicates that this mutation of WEE2 was pathogenic only in the homozygous mutation state. It was predicted that the homozygous mutation(c.T1199A:p.L400Q) in WEE2 might change the kinase activity of Wee 2 and decrease the phosphorylation of $\mathrm{Cdc} 2$, thus reducing the regulation of the inhibition of MPF, and finally lead to the repeated fertilization failure of the proband. The study not only identifies the cause of the infertility, but also expanded the mutation spectrum of WEE2 that caused repeated fertilization failure. Through clinical treatment combined with genetic diagnosis, the cause of partial infertility can be determined, which can timely provide patients with better fertility treatment guidance.

\section{Abbreviations}

IVF: in vitro fertilization; ICSI: intracytoplasmic sperm injection; WEE2: WEE1 homolog 2; GV: germinal vesicle; MPF: maturation promoter factor; Cdc2: Cell Division Cycle 2; CDK1: Cyclin Dependent Kinase 1; MII: metaphase II; RICSI: rescue intracytoplasmic sperm injection; WES: whole exome sequencing; IUI: intrauterine insemination; FQ: Fastaq; ExAC: Exome Aggregation Consortium; PCR: polymerase chain reaction; PN: pronucleus; PB: polar body.

\section{Declarations}

Ethics approval and consent to participate

This study has been approved by the Ethics Committee of Nanjing Drum Tower Staff, and we have obtained the informed consent of all participants in this study. 
Not applicable

Availability of data and material

The datasets during and/or analysed during the current study available from the corresponding author on reasonable request.

Competing interests

The authors declare that they have no competing interests.

\section{Funding}

This study was supported by the National Natural Science Foundation of China (82001494), and the China Postdoctoral Science Foundation (2020M681557).

Authors' contributions

Wenwen Liu, Guijun Yan and Haixiang Sun designed the study. Ningyuan Zhang, Na Kong and Junshun Fang provided clinical sample data, doctor-patient communication, and assessments of patient oocyte retrieval. Min Wu participated in sample collection and processing. Wenwen Liu, Guijun Yan and Ningyuan Zhang analyzed the data and wrote the manuscript. Haixiang Sun directed the research of the subject. All authors read and approved the final manuscript.

Acknowledgements

Not applicable.

Authors' information

${ }^{1}$ Reproductive Medicine Center, The Affiliated Drum Tower Hospital of Nanjing University Medical School, Nanjing 210008, People's Republic of China. 


\section{Reference}

1. Plachot, M. and J. Mandelbaum, Oocyte maturation, fertilization and embryonic growth in vitro. Br Med Bull, 1990. 46(3): p. 675-94.

2. Georgadaki, K., et al., The molecular basis of fertilization (Review). Int J Mol Med, 2016. 38(4): p. 979-86.

3. Esfandiari, N., et al., Complete failed fertilization after intracytoplasmic sperm injection-analysis of 10 years' data. Int J Fertil Womens Med, 2005. 50(4): p. 187-92.

4. Sang, Q., et al., Homozygous Mutations in WEE2 Cause Fertilization Failure and Female Infertility. Am J Hum Genet, 2018. 102(4): p. 649-657.

5. Dai, J., et al., New biallelic mutations in WEE2: expanding the spectrum of mutations that cause fertilization failure or poor fertilization. Fertil Steril, 2019. 111(3): p. 510-518.

6. Yang, X., et al., Homozygous missense mutation Arg207Cys in the WEE2 gene causes female infertility and fertilization failure. J Assist Reprod Genet, 2019. 36(5): p. 965-971.

7. Zhang, Z., et al., Novel mutations in WEE2: Expanding the spectrum of mutations responsible for human fertilization failure. Clin Genet, 2019. 95(4): p. 520-524.

8. Zhao, S., et al., Novel WEE2 gene variants identified in patients with fertilization failure and female infertility. Fertil Steril, 2019. 111(3): p. 519-526.

9. Zhou, X., et al., Novel compound heterozygous mutations in WEE2 causes female infertility and fertilization failure. J Assist Reprod Genet, 2019. 36(9): p. 1957-1962.

10. Wang, A., et al., Clinical exome sequencing identifies novel compound heterozygous mutations of the WEE2 gene in primary infertile women with fertilization failure. Gynecol Endocrinol, 2021: p. 1-6.

11. Tian, Y., et al., Novel compound heterozygous mutation in WEE2 is associated with fertilization failure: case report of an infertile woman and literature review. BMC Womens Health, 2020. 20(1): p. 246.

12. Lew, D.J. and S. Kornbluth, Regulatory roles of cyclin dependent kinase phosphorylation in cell cycle control. Curr Opin Cell Biol, 1996. 8(6): p. 795-804.

13. Han, S.J., et al., Wee1B is an oocyte-specific kinase involved in the control of meiotic arrest in the mouse. Curr Biol, 2005. 15(18): p. 1670-6.

14. Oh, J.S., A. Susor, and M. Conti, Protein tyrosine kinase Wee1B is essential for metaphase // exit in mouse oocytes. Science, 2011. 332(6028): p. 462-5.

15. Jubb, H.C., et al., Arpeggio: A Web Server for Calculating and Visualising Interatomic Interactions in Protein Structures. J Mol Biol, 2017. 429(3): p. 365-371.

16. Pires, D.E., D.B. Ascher, and T.L. Blundell, mCSM: predicting the effects of mutations in proteins using graph-based signatures. Bioinformatics, 2014. 30(3): p. 335-42.

17. Coticchio, G., et al., Focused time-lapse analysis reveals novel aspects of human fertilization and suggests new parameters of embryo viability. Hum Reprod, 2018. 33(1): p. 23-31.

18. Clift, D. and M. Schuh, Restarting life: fertilization and the transition from meiosis to mitosis. Nat Rev Mol Cell Biol, 2013. 14(9): p. 549-62.

19. Rubino, P., et al., The ICS/ procedure from past to future: a systematic review of the more controversial aspects. Hum Reprod Update, 2016. 22(2): p. 194-227.

20. Pan, B. and J. Li, The art of oocyte meiotic arrest regulation. Reprod Biol Endocrinol, 2019. 17(1): p. 8. 
Figure 1. Morphology of a normal zygote and a 2PB oocyte of the proband.

A. 2PN from a normal fertilized oocyte.

B. 2PB oocyte on day 1 after RCISC from the proband. Scale bar, $25 \mu \mathrm{m}$.

The white arrows represent the pronuclei and the black arrows represent the polar bodies.

Figure 2. Identification of WEE2 mutations in proband diagnosed with repeated fertilization failure.

A. Family pedigree of proband with repeated fertilization failure. Squares and circles represent male and female members, respectively. The black entity symbol represents the affected subject, the open symbol represents the unaffected subject, and the equal sign represents sterility. The black arrows indicates the female proband.

B. Sanger sequencing results of WEE2 mutation identification. The proband (II-1) was identified as a homozygous mutation of WEE2. Her father (I-1) did not carry the mutation, her mother (I-2) was a heterozygous mutation, and her two sisters (II-3,II-4) were also mutation carriers. All the three women with heterozygous mutation had normal fertility. The black arrows indicate the mutant deoxynucleotide bases in the WEE2 sequence.

Figure 3. Position and conservation of WEE2 mutation c.T1199A:p.L400Q.

A. Loaction of the mutation in the genomic structure and its protein structure. The mutation is located in exon 8 of WEE2 gene structure, and the black arrow indicates the location of c.T1199A. The mutation was located in the Wee2 Pkinase domain, as indicated by the circular arrow.

B. Mutation p.L400Q of Wee2 was conserved in several different species.

Figure 4. Prediction of protein structure and stability changes of the Wee2 mutation L400Q.

The online software Arpeggio was used to analyze the interatomic interactions in mutation of leucine at position 400 to glutamine of protein, and the mCSM online service was used to predict the effect of mutations in protein. The red dashed box indicates the interatomic interactions in mutation of leucine at position 400. 


\section{Figures}

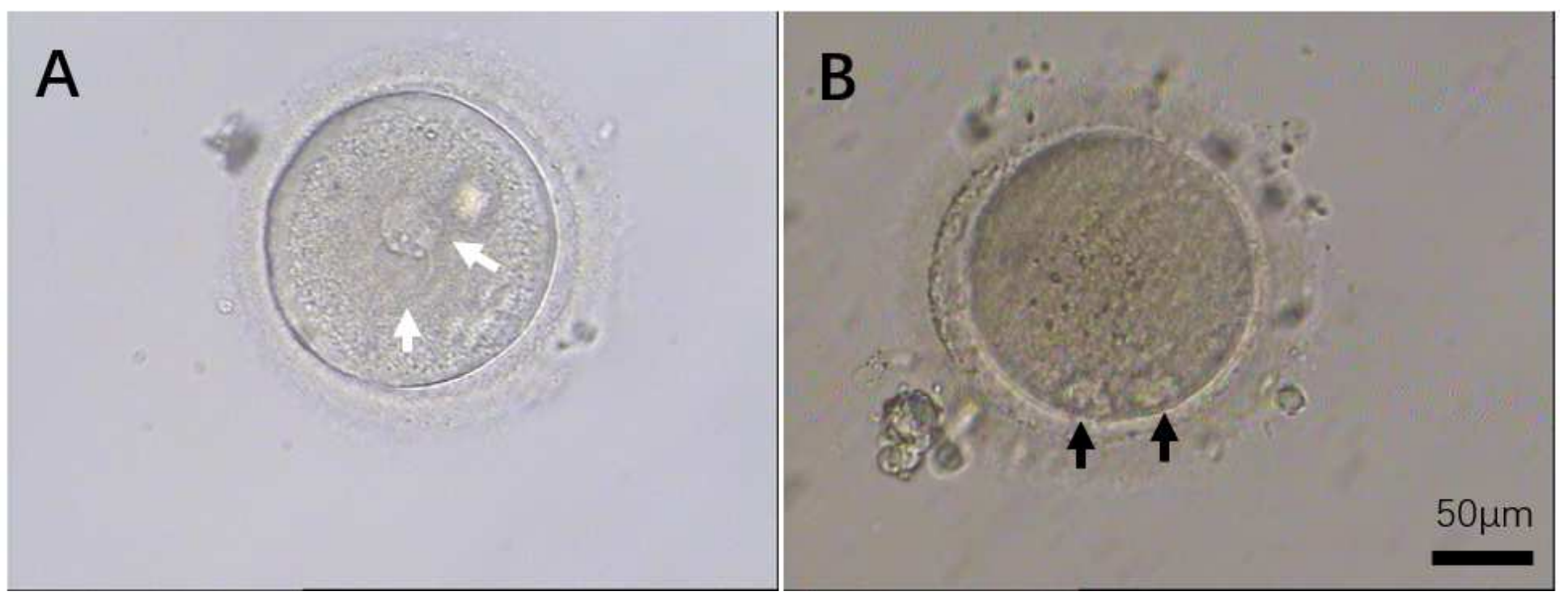

\section{Figure 1}

Morphology of a normal zygote and a 2PB oocyte of the proband. A. 2PN from a normal fertilized oocyte. B. 2PB oocyte on day 1 after RCISC from the proband. Scale bar, $25 \mu \mathrm{m}$. The white arrows represent the pronuclei and the black arrows represent the polar bodies. 
A

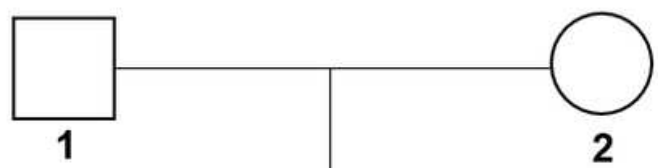

WT/WT

L400Q/ WT

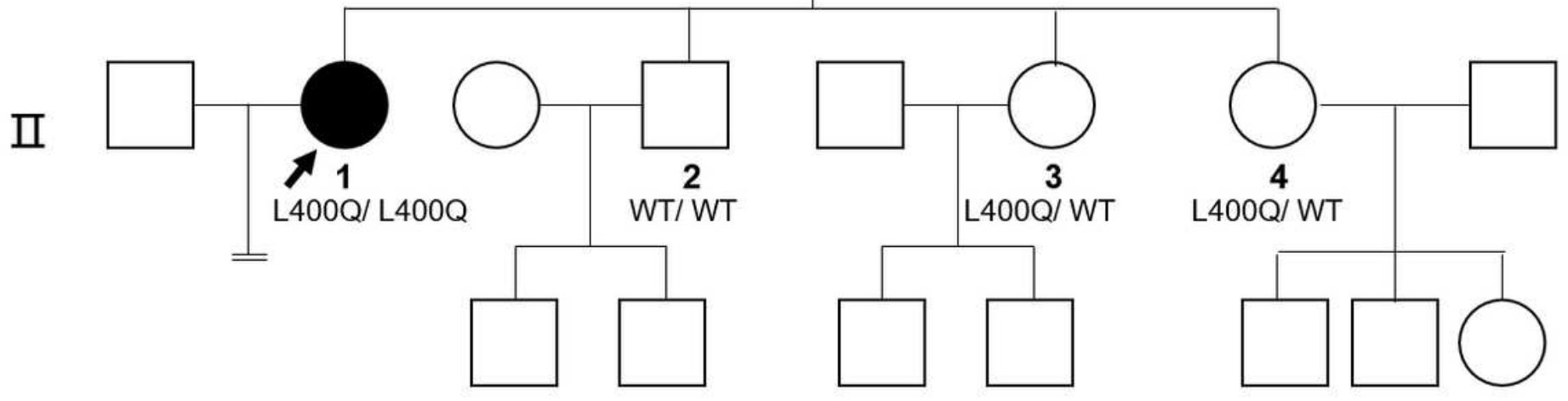

B

I -1

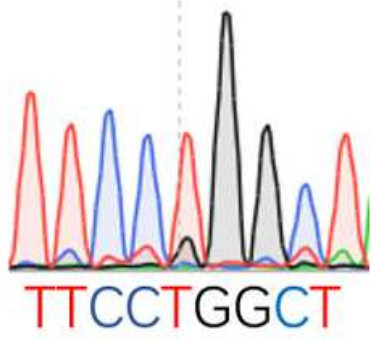

П-2

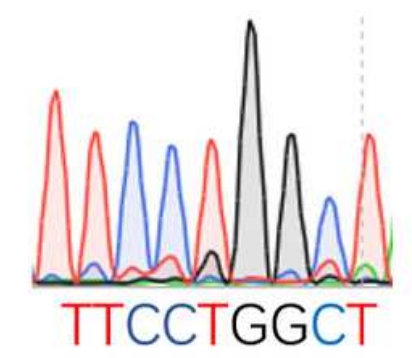

I -2

c.T1199A:p. L400Q

ㅍ-3

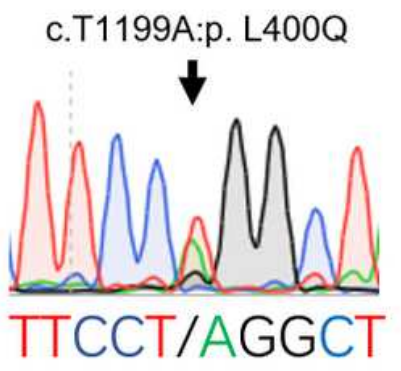

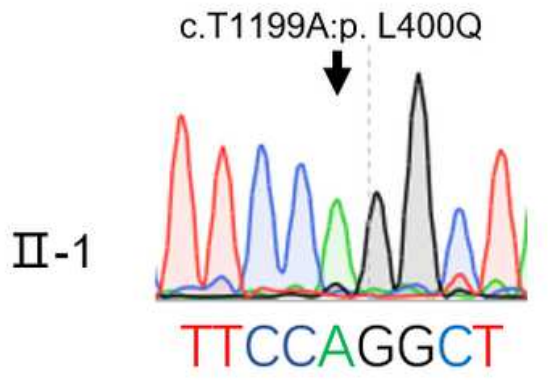

П-4

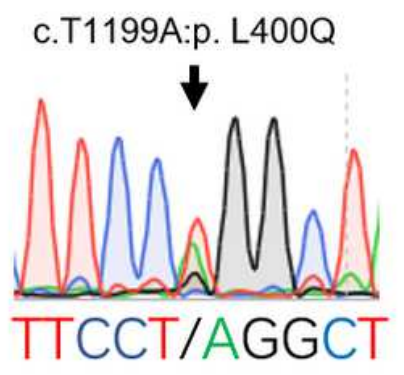

Figure 2

Identification of WEE2 mutations in proband diagnosed with repeated fertilization failure. A. Family pedigree of proband with repeated fertilization failure. Squares and circles represent male and female members, respectively. The black entity symbol represents the affected subject, the open symbol represents the unaffected subject, and the equal sign represents sterility. The black arrows indicates the female proband. B. Sanger sequencing results of WEE2 mutation identification. The proband (II-1) was identified as a homozygous mutation of WEE2. Her father (I-1) did not carry the mutation, her mother (I-2) was a heterozygous mutation, and her two sisters (II-3,II-4) were also mutation carriers. All the three women with heterozygous mutation had normal fertility. The black arrows indicate the mutant deoxynucleotide bases in the WEE2 sequence 
A

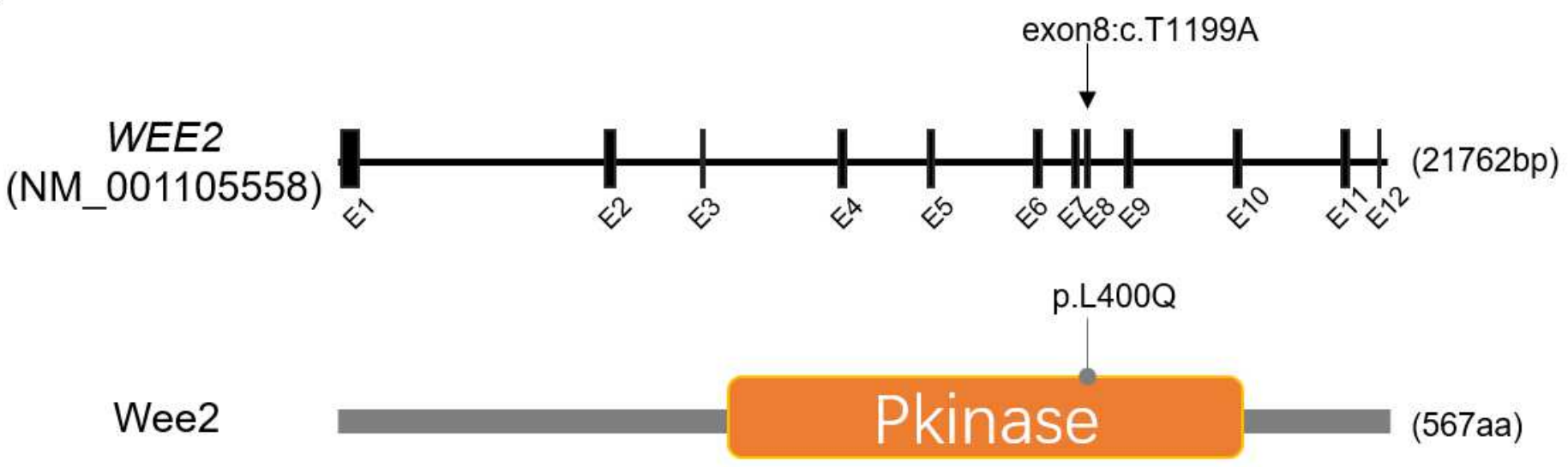

B

Homo sapiens

Pan troglodytes

Macaca mulatta

Canis lupus familiaris

Bos taurus

Mus musculus

Rattus norvegicus

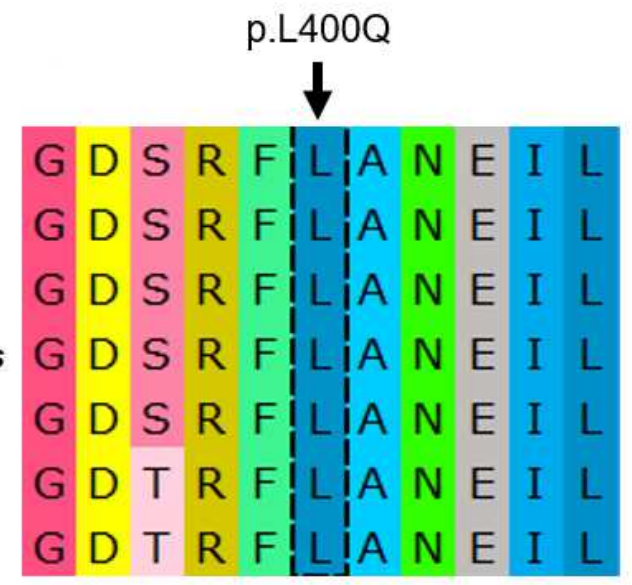

\section{Figure 3}

Position and conservation of WEE2 mutation c.T1199A:p.L400Q. A. Loaction of the mutation in the genomic structure and its protein structure. The mutation is located in exon 8 of WEE2 gene structure, and the black arrow indicates the location of c.T1199A. The mutation was located in the Wee2 Pkinase domain, as indicated by the circular arrow. B. Mutation p.L400Q of Wee2 was conserved in several different species. 


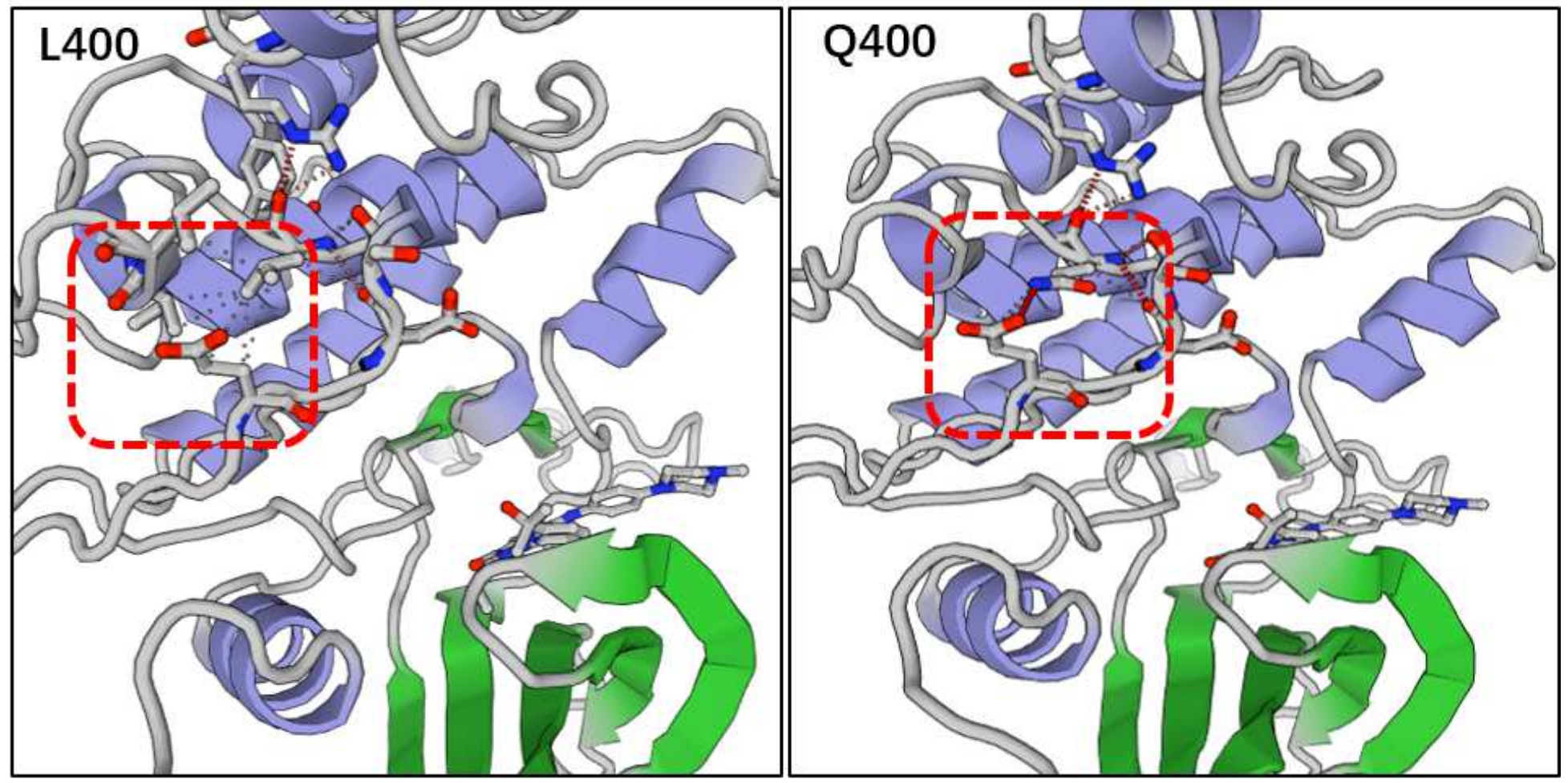

Predicted Stability Change $(\Delta \Delta \mathbf{G})$ :

$-1.604 \mathrm{kcal} / \mathrm{mol}$ (Destabilizing)

Mutation:

Wild-type: $\mathbf{L}$

Position: $\mathbf{4 0 0}$

Mutant-type: Q

Chain: A

\section{Figure 4}

Prediction of protein structure and stability changes of the Wee2 mutation L400Q. The online software Arpeggio was used to analyze the interatomic interactions in mutation of leucine at position 400 to glutamine of protein, and the mCSM online service was used to predict the effect of mutations in protein. The red dashed box indicates the interatomic interactions in mutation of leucine at position $\mathbf{4 0 0}$

\section{Supplementary Files}

This is a list of supplementary files associated with this preprint. Click to download.

- Table2.png

- Table1.png 